min) compared with subjects not having AMS (74.30\% \pm $5.48 \%$ and $79.80 \pm 9.9$ beats/min, respectively), but no correlation with IOP was noted in regard to AMS.

Conclusions.-No significant change of IOP was noted during high altitude exposure over 4 days spent at high altitude compared with baseline. However, a tendency for a decreased IOP during high altitude exposure was noted. No correlation between IOP and AMS scores or clinical parameters was observed.

Gabriel Willmann, MD

Andreas Schatz, MD

M. Dominik Fischer, MD

Eberhart Zrenner, MD, PhD

Karl U. Bartz-Schmidt, MD

Florian Gekeler, MD

Tübingen, Germany

Kai Schommer, MD

Heidelberg, Germany

\section{Hypoxia in the Eye-Retinal Changes in Correlation to Acute Mountain Sickness}

Background.-The visual system undergoes profound changes when exposed to hypoxia or high altitude. Although some of these changes, such as increased blood flow and increased tortuosity of blood vessels, present physiological adaptive mechanisms to high altitude exposure, others present potentially pathological changes due to hypoxia, for example, optic disc swelling or retinal hemorrhages, which in part have previously been correlated with AMS.

Objective.-These visual system changes are of special interest or study as the retina is a directly visible part of the central nervous system, and thus may be able to give insight into a possible underlying pathologic mechanism of AMS.

Methods.-With a long history of high altitude research focusing on ocular changes during mountaineering expeditions, the Tübingen High Altitude Ophthalmology study investigated retinal changes in 14 healthy subjects during acute high altitude exposure in a controlled study setting at the Capanna Margherita (Italy) at $4559 \mathrm{~m}$ using state-of-the-art equipment to study morphological and functional changes of the retina in regard to AMS. Retinal function was assessed in psychophysical tests and in objective measurements such as microperimetry (MP1, Nidek, Japan). Morphological changes using spectral domain optical coherence tomography, fluorescein angiography, and Heidelberg retinal angiography (Spectralis optical coherence tomography plus Heidelberg retinal angiograph and Heidelberg retinal tomograph, Heidelberg Engineering, Heidelberg, Germany]) during acute high altitude exposure were tested for the first time.

Results.-Fluorescein angiography showed considerable dye leakage in the periphery of the retina and increased vessel diameters. Optical coherence tomography and Heidelberg retinal tomograph measurements demonstrated swelling of the optic disc as well as distinct foveal changes with perifoveal thickening of the retinal nerve fiber layer and vascular arcades.
Conclusions.-No correlation between these changes and central retinal function and AMS was found.

Gabriel Willmann, MD

Andreas Schatz, MD

M. Dominik Fischer, MD

Eberhart Zrenner, MD, PhD

Karl U. Bartz-Schmidt, MD

Florian Gekeler, MD

Tübingen, Germany

Kai Schommer, MD

Heidelberg, Germany

\section{Burning Man 2011: Mass Gathering Medical Care in a Hostile and Austere Environment}

Background.-Burning Man is a weeklong outdoor arts festival held annually on a dry Pleistocene lakebed in the rugged and austere Black Rock Desert Wilderness Area in northern Nevada. The 2011 event presented numerous challenges in the provision of emergency medical services and medical care owing to attendance size, the hostile environment, and distance to definitive medical care.

Objective.-Our purpose was to describe the planning and implementation of medical care at a mass gathering event in a harsh and austere wilderness environment. This was a retrospective, observational review of the preparation, management, and implementation of medical care.

Methods.-Total attendance was 53 375, with a total of 2307 patients. Emergency medical services operations included 6 ambulances, 3 quick response vehicles, 2 first aid stations, and a physician staffed mobile hospital. The hospital had limited diagnostic capabilities and a limited formulary. Most conditions treated were minor, the most common being soft tissue injuries, dehydration, eye problems, and urinary tract infections. Most patients were treated and released. A total of 33 patients was transferred out. Several significant cases were seen, including a patient with a spontaneous subarachnoid hemorrhage who later died; there were several cases of trauma, a traumatic pneumothorax, and a thoracic aortic dissection. The latter patient sustained a cardiac arrest at the event, was resuscitated, flown to Reno, underwent emergency surgery, and survived to discharge. A unique phenomenon called "playa foot," due to an alkali burn of the feet from constant exposure to the dry lake bed dust, was frequently encountered.

Results.-Overall, Burning Man 2011 was successful. The goal of providing the highest level of care possible in an austere environment and allowing as many patients as possible to remain at the event was achieved.

Conclusions.-Our experience can help guide others planning for similar events under similar austere conditions in the future.

Ryan Hodnick, DO Bryan E. Bledsoe, DO Kelly Buchanan, MD

Jeff Westin, MD

Loren Gorosh, MD

Las Vegas, NV, USA 
Pat Songer, NREMT-P Winпетисса, NV, USA

\section{The Who and Why of Wilderness Medicine Conference Attendance}

Background.-The origin of wilderness medicine is unclear and depends on where you begin. Growth in the field, however, is clear, particularly since the 1980s. For example, the Wilderness Medicine Institute has trained more than 88,000 students since starting in 1990.

Objective.-This short study attempted to elucidate why people attend the new wilderness medicine conferences, what influences their decision to attend a conference, and who is it that attends them.

Methods.-A voluntary, blinded survey was distributed at a local wilderness medicine conference with approximately 100 attendees. Thirty-nine attendees responded to the survey.

Results.-Of the respondents, $84.6 \%$ were in medical careers $(8$ emergency medical technicians, 6 physicians, 5 registered nurses, 3 medical students, 3 paramedics, 4 nurse practitioner/physician assistants, and 4 others). When people ranked the top 3 reasons for attending a wilderness medicine conference, a desire to know more for personal academic reasons accounted for $28.2 \%$ of responses, and personal medical reasons (ie, family and personal trips and hobbies might involve wilderness injuries) accounted for $24.5 \%$ of responses, followed by continuing medical education credits (13.6\%), meeting people with similar interests $(11.8 \%$ ), professional (Ski Patrol, SAR, and so forth [10.9\%]), and Fellow of the Academy of Wilderness Medicine credits (8.2\%). When asked to give the top 3 reasons why they picked this conference in particular, $27.4 \%$ said location, $15.9 \%$ said timing, $15.9 \%$ said the organization/director, and $15 \%$ said the price.

Conclusions.-More than half of people attend wilderness conferences for personal reasons, but more than 35\% also attend for continuing medical education, professional, or Fellow of the Academy of Wilderness Medicine credits. Location, timing, price, and the organizer accounts for a large factor in selecting a particular conference, and most people who attend have a significant medical background. Surveys such as this help explain why people are so interested in wilderness medicine and may help to guide the planning of the conferences.

Richard W. Trierweiler, MD, MPH York, PA, USA

\section{Rates of Motorcycle-Animal Collisions in the Midwest}

Background.-Motorcycle-animal collisions have been described in several small case series. The true burden of these collisions remains unknown.

Objective.-The aim of this study is to determine rates of injury and fatality among motorcycle-animal collisions in the Midwest.

Methods.-A retrospective cohort of motorcycle-animal collisions occurring from 2004 to $2010(n=4941)$ was created from existing data extrapolated from the Michigan Traffic Crash Facts Database, the Wisconsin Department of Transportation, and the Minnesota and Ohio Departments of Public Safety. Motorcycle registrations were collected from the Federal Highway Administration. Estimates of deer populations were collected from the respective state Departments of Natural Resources.

Results.-A total of 184 fatal (Michigan 25.5\%, Wisconsin $34.2 \%$, Minnesota 16.3\%, and Ohio 23.9\%) and 4757 nonfatal (Michigan 45\%, Minnesota 17\%, and Ohio 38\%) motorcycleanimal collisions were reported between 2004 and 2010. These states represent $16.2 \%$ of all motorcycle registrations in the United States, and that number has increased each year. Deer populations have a nonsignificant downward trend (5.32 to 4.97 million). Overall collision rates per 100000 registered motorcycles have not changed (81.1 to 73.0). Among individual states, collision rates decreased in Minnesota (64.2 to 46.3, $P=$ $.002, R^{2}=0.87$ ), and Michigan had the highest mean collision rate (121.7, SE 10.5). Total injuries (79.2\%) and fatalities $(3.22 \%)$ were equivalent to nonanimal-motorcycle collisions (79.4\% and 3.58\%, respectively). Injury rates per 100 collisions have remained stable (77.1 to 79.8). Fatalities rates per 100 collisions have a nonsignificant downward trend in Ohio and upward trends in Minnesota and Michigan. The overall mean fatality and injury rates per 100 collisions are 3.8 (SE 0.25) and 79.2 (SE 1.25), respectively.

Conclusions.- Rates of motorcycle-animal collisions, fatalities, and injuries have remained stable in the Midwest. Collision rates have decreased in Minnesota, but injury and fatality rates have upward trends. Further efforts to decrease interactions between animals and motorcycles should be pursued.

Nathaniel T. Hibbs, DO

East Lansing, MI, USA

Dave Betten, MD

Lansing, MI, USA

\section{Fatalities and Injuries Due to Motorcycle-Deer Collisions} in Michigan

Background.-Motorcycle-deer collisions have been described in several small case series. Risk factors for motorcycle-deer collisions and opportunities for intervention have yet to be described.

Objective.-The aim of this case-control study is to determine risk factors associated with fatal and injury-related motorcycle-deer collisions.

Methods.-The study group was created from Michigan State Police reports and were abstracted from the Michigan Traffic Crash Facts Database for motorcycle-deer collisions occurring from 2004 to $2011(\mathrm{n}=2692)$.

Results.-A total of 50 fatal (1.9\%) and 2642 nonfatal $(98.1 \%)$ collisions were reported during the study period. Persons involved in fatal collisions were older than those in nonfatal collisions (54.3 vs $44.3, P=.028$ ). Injury severity was evenly distributed (20.3\% incapacitating, $31.7 \%$ nonincapacitating, $22.2 \%$ possible injury, and $25.8 \%$ not injured). Transfer to hospital was highest among persons with incapacitating 